\title{
saguf
}

schweizerische akademische gesellschaft für

umweltforschung und ökologie

societé académique suisse pour la recherche

sur l'environnement et l'écologie

swiss academic society for

environmental research and ecology

\section{Die Energienachfrage steuern Handlungsempfehlungen zur Nutzung von Smart-Meter-Daten}

Mit digitalen Stromzählern - smart meter - können Stromkund(inn)en zu energieeffizientem Verhalten motiviert werden. Mit Expert(inn)en aus Forschung und Praxis hat die saguf-Arbeitsgruppe Energiezukunft Handlungsempfehlungen zur Nutzung von SmartMeter-Daten sowie zum optimierten Stromverbrauch erarbeitet.
Vicente Carabias, Ursula Eschenauer, Tobias Kuehn, Evelyn Lobsiger-Kägi, Thoralf Mildenberger

Regulating Energy Demand. Recommendations for the Use of Smart Meter Data and Demand Side Management GAIA 25/3 (2016): 217-219 | Keywords: behavioural change, demand side management, energy consumption, energy transition

$\mathrm{D}$ ie Energiestrategie 2050 der Schweiz gibt ambitionierte Ziele vor, den Gesamtenergiebedarf $\mathrm{zu}$ reduzieren und erneuerbare Energieträger auszubauen (wie etwa Photovoltaik und Windkraft). Dieser Ausbau wird von der heutigen nachfragegesteuerten Stromproduktion zum künftig angebotsgesteuerten Stromverbrauch führen, da die Stromproduktion aus Sonnen- und Windenergie wetterabhängig ist. Diese Umstellung erfordert nicht nur die Bereitstellung von Reserveenergien und Speichertechnologien, sondern auch erhöhtes Stromsparen und zeitliche Stromlastverschiebungen durch die Einführung von smart grids. Daher werden smart meter (Abbildung 1, S. 218) ein zentraler Bestandteil dieser zukünftigen Stromnetze sein: So können die digitalen Stromzähler mit Rückmeldungen zum aktuellen Stromverbrauch die Endverbraucher(innen) zum Stromsparen und zur Lastverschiebung motivieren (Tampe-Mai und Mack 2016); zudem ermöglicht der Einsatz von smart meter die automatische Laststeuerung und das Demand-Side-Management (DSM) durch Energieversorger und -dienstleister.

\section{Das Power Saver Café}

Die saguf-Arbeitsgruppe Energiezukunft organisierte - vor dem Hintergrund eines Pilotprojekts der Zürcher Hochschule für
Angewandte Wissenschaften (ZHAW) und Stadtwerk Winterthur zur Untersuchung von Smart-Meter-Daten in Abhängigkeit von sozio-ökonomischen Faktoren (zum Beispiel Haushaltsgröße und -art, steuerbares Einkommen) in drei Quartieren der Stadt Winterthur - im Dezember 2015 einen Stakeholder-Workshop. Bei diesem wurde über einen erleichterten Datenzugang und die Frage, wie Haushalte für einen sparsameren und zeitlich flexibleren Energieeinsatz sensibilisiert werden können, diskutiert (Carabias et al. 2015). An dem transdisziplinär ausgerichteten Workshop nahmen, wie in einem früheren saguf-Beitrag empfohlen (Carabias-Hütter et al. 2013), Mitglieder der AG Energiezukunft sowie weitere Expert(inn)en aus der Forschung (Energiewirtschaft, Umweltnaturwissenschaften, Umweltpsychologie) und Stakeholder aus der Praxis (Bundesamt für Energie, Energieversorgungsunternehmen [EVU], Stadtverwaltung) teil. Zu Beginn des Workshops wurden die Ergebnisse aus den Pilotstudien mit Smart-Meter-Daten und zu DSM vorgestellt. In einem Power Saver Café über vier Runden diskutierten die Teilnehmenden danach folgende Themen:

- Beschaffung, Zusammenführung und Analyse von Smart-Meter- sowie weiteren relevanten sozio-ökonomischen Daten,
- Demand-Side- und Lastgang-Management,

- bedarfsangepasste Produkt-/Dienstleistungsangebote für VerbraucherCluster und

- Interventionen und Kampagnen für den sparsamen und zeitlich flexiblen Stromverbrauch.

Die Diskussionen an den Tischen wurden jeweils von einer Person moderiert und protokolliert. Die Workshopteilnehmenden sollten Handlungsempfehlungen anhand vorbereiteter Fragen diskutieren und die zuständigen Akteure benennen. Anschließend glich das Projektteam die Ergebnisse mit ähnlichen Vorschlägen aus der Literatur ab und ergänzte sie. Im Fol-

Kontakt Autor(inn)en: Vicente Carabias | saguf | Vorstand und Arbeitsgruppe Energiezukunft | Zürcher Hochschule für Angewandte Wissenschaften (ZHAW) | Institut für Nachhaltige Entwicklung | Winterthur | Schweiz | E-Mail: cahu@zhaw.ch | www.smartcitywinterthur.ch

Kontakt saguf: saguf-Geschäftsstelle | Dr. Claudia Zingerli | ETH Zentrum CHN | 8092 Zürich | Schweiz |E-Mail: saguf@env.ethz.ch|www.saguf.ch

(C) 2016 V. Carabias et al.; licensee oekom verlag. This is an article distributed under the terms

of the Creative Commons Attribution License (http://creativecommons.org/licenses/by/3.0)

which permits unrestricted use, distribution, and reproduction in any medium, provided the original work is properly cited. 
genden sind die wichtigsten Ergebnisse aus den vier Bereichen zusammengefasst (alle Ergebnisse finden sich in Eschenauer et al. 2016).

\section{Datenbeschaffung und -zusammen- führung}

Die vorhandene Datenbasis der Stadt Winterthur wurde im Rahmen der Pilotstudie Stromverbrauchsanalyse durch die Informatikdienste Winterthur mit derjenigen des Stadtwerks Winterthur verknüpft und anonymisiert. So kombinierten wir Stromverbrauchsmuster von 257 Privathaushalten und 35 Gewerbebetrieben aus drei verschiedenen Quartieren beziehungsweise Wohnsiedlungen mit sozio-ökonomischen Daten. Aus Datenschutzgründen mussten die Daten teilweise aufwendig manuell zusammengeführt werden. Darauf aufbauend wurden im Workshop folgende Handlungsempfehlungen für die Datenverknüpfung erarbeitet:

- In der Stadtverwaltung sollten Datenbestände entweder zentral erfasst werden, etwa in einem Data-Warehouse, oder es sollte eine Einheit bestimmt werden, die einen Überblick über die Datenbestände hat.
- Der Prozess der Datenerhebung/-zusammenführung sollte standardisiert und entsprechend dokumentiert werden, unter anderem hinsichtlich Herkunft der Rohdaten, Jahrgang und Aktualisierungshäufigkeit der Statistiken. Dies sollte in der Stadtverwaltung, bei EVUs oder weiteren Dateneigentümern erfolgen.

\section{Demand-Side- und Lastgang- Management}

In ihrem Kurzinput stellte Nina Boogen von der ETH Zürich das vom Bundesamt für Energie geförderte Projekt An Evaluation of the Impact of Energy Efficiency Policies on Residential Electricity Demand in Switzerland vor, das einen reduzierenden Einfluss von DSM-Programmen von EVU auf den Elektrizitätsverbrauch von Haushalten festgestellt hatte. Daher wurden im Power Saver Café weitere Optionen von DSM und Lastgang-Management diskutiert.

Bei den Expertendiskussionen über die Möglichkeiten zur Lastverschiebung wurde deutlich, dass die automatische Laststeuerung ohne Komforteinbuße und Kostenfolgen als am erfolgversprechendsten gilt. Deshalb fokussieren die folgenden
Handlungsempfehlungen auf diesen Bereich (zuständige Akteure in Klammern):

- Geeignete Geräte für Lastverschiebung sind Wäschetrockner, Spülmaschine und Herd, da sie am meisten zu den Verbrauchspeaks beitragen (McLoughlin et al. 2012). Es sollten daher Möglichkeiten aufgezeigt werden, wie diese Geräte zu anderen Zeiten eingesetzt werden können. (EVU)

- Eine automatische Laststeuerung sollte das EVU bei bestimmten Geräten als Standard voreinstellen (default), wobei die Möglichkeit der manuellen Steuerung anzubieten wäre. (EVU)

- Falls dynamische Preise von EVUs angeboten werden, ist es ratsam, mit vorhersagbaren, einfachen, transparenten Angeboten zu beginnen. Mit steigender Komplexität der Tarife sollten diese mit zusätzlichem Service und Produkten ergänzt werden (Dütschke und Paetz 2013). (EVU)

\section{Produkt-/Dienstleistungsangebote für spezifische Cluster}

Wir hatten die Stromverbrauchsdaten mit einer statistischen Cluster-Analyse ausgewertet, um Gruppen von Haushalten mit

ABBILDUNG 1: Mit smart meter kann der Stromverbrauch gesenkt werden - über Displays, Webportale oder Smartphone-Apps geben diese intelligenten Stromzähler den Stromkund(inn)en Feedback zu ihrem Stromverbrauch.

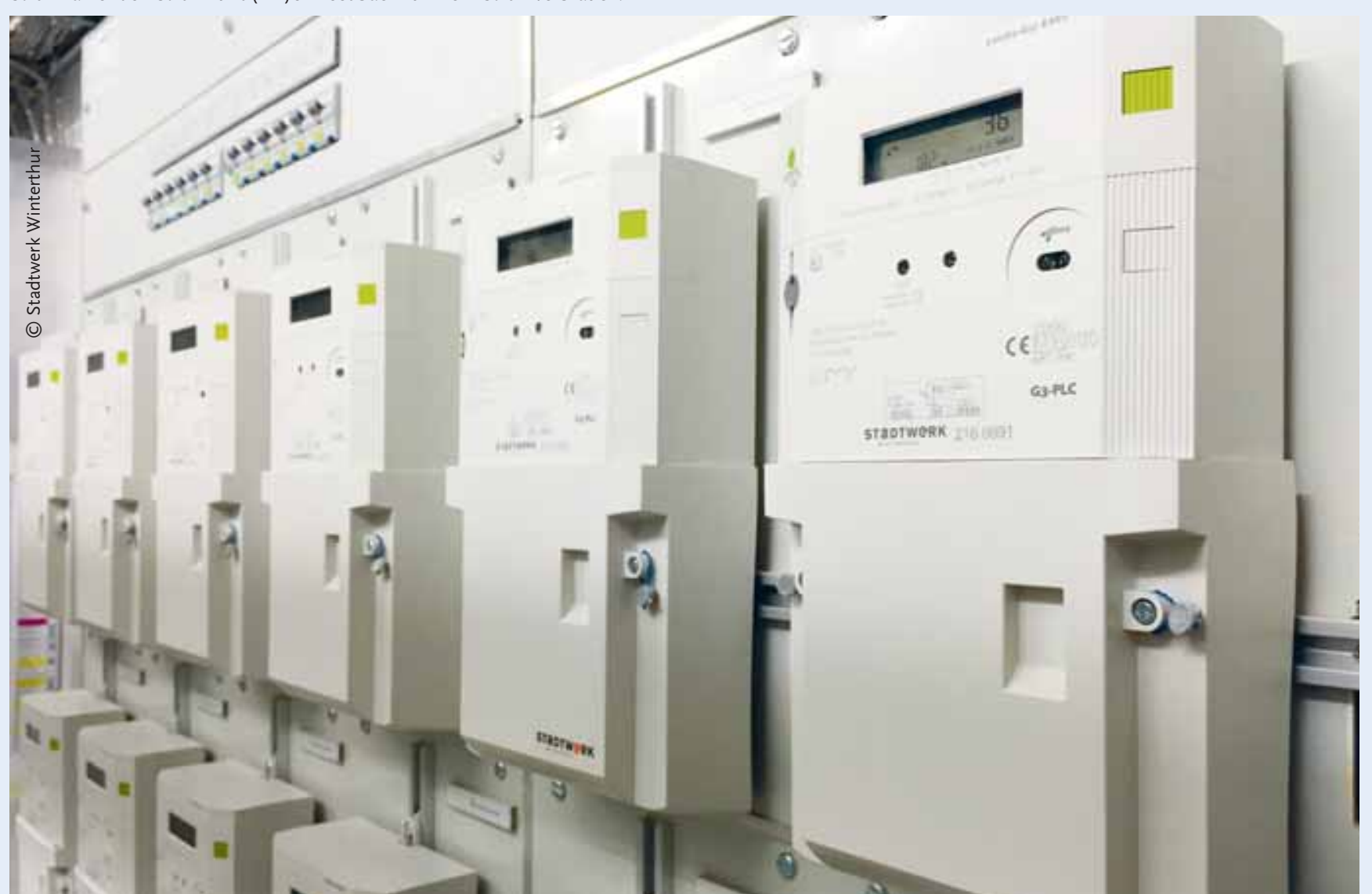


jeweils ähnlichen Verbrauchsmustern zu eruieren. Da die Messstellen eines Clusters hinsichtlich Anzahl und Alter der Bewohner(innen) sowie Bruttofläche und Einkommen unterschiedlich sind, ergeben sich folgende Handlungsempfehlungen: - Eine zielgruppenspezifische Auswertung der Smart-Meter-Daten könnte für die Bestimmung geeigneter Interventionen sowie als Kontroll- und Planungstool hilfreich sein. (Forschung)

- EVUs wollen ihre Kund(inn)en für ökologische Stromprodukte gewinnen und erreichen dies oft mit defaults für ein ökologisches Basisprodukt. ${ }^{1}$ (EVU)

Durch den alleinigen Einsatz von smart meter werden Stromverbraucher(innen) kaum dazu motiviert, langfristig Energie im Haushalt einzusparen. Vielmehr ist ein zusätzliches, aktives und personalisiertes Feedback erfolgversprechend. Verbraucher(innen) können beispielsweise zum Stromsparen angeregt werden, indem sie ihren Stromverbrauch mit dem des Vorjahres und anderen Haushalte vergleichen. Zudem könnten Onlineplattformen und Apps Tipps, Analysen und Beratungen anbieten (Tampe-Mai und Mack 2016). Und Gamification-Elemente führen dazu, dass sich die Haushalte stärker mit der Thematik auseinandersetzen (Castri et al. 2016).

Eine weitere Idee zum Energiesparen sind innovative Serviceleistungen, die das Teilen und das Leasing von Produkten ermöglichen, wie der Service Zehn Jahre lang ein warmes Haus statt Neuanschaffung einer Heizung. Zudem sollten smarte Technologien und Automatisierung Geräte in Zukunft effizienter machen.

\section{Interventionen und Kampagnen}

Zusätzlich diskutierten die Teilnehmenden) allgemeine Energiespartipps, die unabhängig vom Gebrauch von smart meter umgesetzt werden könnten, etwa in Form von Interventionen und Kampagnen. Folgende Anregungen können hilfreich sein:

- Bei Kampagnen ist es sinnvoll, mit wenigen, immer gleichen Botschaften $\mathrm{zu}$ arbeiten (siehe zum Beispiel die gelungene PET-Recycling-Kampagne des Vereins PET-Recycling Schweiz ${ }^{2}$ mit klarem Fokus auf einen Flaschentyp) und dabei wirkungsvolle und einfach umzusetzende Maßnahmen zu verwenden. (Verbände, EVU, Bund, Kommunikationsfachstellen)

- Berühmte Persönlichkeiten gewinnen, die für Energiesparen werben, Schulen einbinden. (Bund, Verbände, Schulen, EVU, Kommunikationsfachstellen)

- Um den Energieverbrauch besser vergleichen zu können, sollten Alltagshandlungen unter die Lupe genommen werden (beispielsweise Licht eine Stunde brennen lassen vs. ein Waschgang bei 90 Grad Celsius). (EVU, Kommunikationsfachstellen)

\section{Fazit}

Da Smart-Meter-Daten momentan oft nur lückenhaft zur Verfügung stehen, können nur schwer kundenspezifische Empfehlungen gegeben werden. Es bedarf einer flächendeckenden Erhebung über einen längeren Zeitraum in einer Region. Eine weitere wichtige Erkenntnis ist, dass künftige DSM-Angebote für Kund(inn)en sehr niederschwellig und ohne große Komforteinbußen gestaltet werden sollten. Fast alle DSM-Maßnahmen wurden generell den EVUs zugeordnet, obwohl Untersuchungen gezeigt haben, dass Stadtverwaltungen als Absender für Energieanliegen oftmals als vertrauenswürdiger angesehen werden (Heinstein et al. 2013). Mit den genannten Handlungsempfehlungen und anstehenden Folgeprojekten (Eschenauer et al. 2016) kann die Thematik weiter vertieft werden und so zu konkreten Umsetzungsschritten hin zu smart grids beitragen. In der saguf-Arbeitsgruppe Energiezukunft werden zudem die damit verbundenen Prozesse der gesellschaftlichen Transformation weiter behandelt.

Der Stakeholder-Workshop wurde von der Akademie der Naturwissenschaften Schweiz (SCNAT) finanziell unterstützt. Für die Durchführung der Stromverbrauchsanalyse in Winterthur danken wir den Praxispartnern von Stadtwerk Winterthur und den verschiedenen Ämtern der Stadt Winterthur sowie dem Bundesamt für Energie für die finanzielle Unterstützung im Rahmen der Initiative Smart City Winterthur.

\section{Literatur}

Carabias, V., T. Mildenberger, T. Kuehn, E. LobsigerKägi, U. Eschenauer. 2015. Stakeholder-Workshop Handlungsempfehlungen zum Teilprojekt Strom-

\section{saguf-Arbeitsgruppe Energiezukunf}

Die saguf-Arbeitsgruppe Energiezukunft beschäftigt sich mit dem Energiewendeprozess und der dazugehörigen Energieforschung. Sie unterzieht diese einer kritischen Reflexion und fragt, ob die maßgeblichen gesellschaftlichen Fragen der Energiezukunft in der Energieforschung enthalten sind.

In Sitzungen unter den Arbeitsgruppenmitgliedern sowie in Workshops mit Energieexpert(inn)en aus Universitäten, Fachhochschulen und weiteren Stakeholdern von Behörden, Wirtschaft und Nichtregierungsorganisationen werden die Themen der Energiestrategien und der Energieforschung gegenübergestellt.

Sind Sie an einer aktiven Teilnahme in der saguf-Arbeitsgruppe Energiezukunft interessiert? Dann melden Sie sich bitte bei den auf Seite 237 genannten Kontaktpersonen.

WEITERE INFORMATIONEN:

www.ez.saguf.ch

verbrauchsanalyse des Forschungsprojekts Smart City Winterthur. Winterthur: Zürcher Hochschule für Angewandte Wissenschaften (ZHAW).

Carabias-Hütter, V., C. Moser, H. Spiess. 2013. Anticipating gaps in energy research at transdisciplinary and international interfaces. GAIA 22/4: 281-283.

Castri, R. et al. 2016. Triggering electricity-saving through smart meters: Play, learn and interact using gamification and social comparison. Proceedings Energy-Feedback Symposium. TEDDINET: Edinburgh. Edinburgh, July 4 to 5. 248-256.

Dütschke, E., A. G. Paetz. 2013. Dynamic electricity pricing: Which programs do consumers prefer? Energy Policy 59: 226-234.

Eschenauer, U., T. Mildenberger, E. Lobsiger-Kägi, T. Kuehn, V. Carabias. 2016. Schlussbericht zum Teilprojekt Stromverbrauchsanalyse des Forschungsprojekts Smart City Winterthur. Winterthur: Zürcher Hochschule für Angewandte Wissenschaften (ZHAW).

Heinstein, P., C. Ballif, L.-E. Perret-Aebi. 2013. Building integrated photovoltaics (BIPV): Review, potentials, barriers and myths. Green 3/2: 125-156.

McLoughlin, F. et al. 2012. Characterising domestic electricity consumption patterns by dwelling and occupant socio-economic variables: An Irish case study. Energy and Buildings 48: 240-248.

Tampe-Mai, K., B. Mack. 2016. Smart meter intelligent nutzen. GAIA 25/2: 135-136.

1 Der Stadt Winterthur wurde empfohlen, das Ökostromprodukt $e$-Strom. Silber als default bei Stromprodukten einzusetzen anstatt wie derzeit noch das weniger ökologische Produkt e-Strom. Bronze. 2 www.petrecycling.ch/de/wissen/zahlen-fakten/fakten 